\section{Temperature-compensated optical fiber Michelson refractometer}

\author{
Adriaan van Brakel, MEMBER SPIE \\ Pieter L. Swart, MEMBER SPIE \\ Rand Afrikaans University \\ Center for Optical Communications and \\ Sensors \\ Faculty of Engineering \\ PO Box 524 \\ Auckland Park 2006 \\ South Africa \\ E-mail: pls@ing.rau.ac.za
}

\begin{abstract}
Fiber optic interferometric refractometers usually possess large temperature sensitivities, especially those based on individual long-period gratings. This work introduces a compound-cavity Michelson interferometer that uses mode coupling in a long-period grating to establish the two optical paths in a single fiber, and therefore presents a compact sensor for in vivo refractive index measurement. Successful operation of the athermal refractometer was demonstrated experimentally by comparing its phase shift due to temperature-dependent changes of the refractive index of the analyte with refractive index readings from a temperature-controlled Abbe refractometer. () 2005 Society of Photo-Optical Instrumentation Engineers. [DOI: 10.1117/1.1850746]
\end{abstract}

Subject terms: fiber optic sensors; refractometry; long-period gratings; Michelson interferometers; temperature compensation; refractive index.

Paper L040467 received Jul. 13, 2004; revised manuscript received Sep. 17, 2004; accepted for publication Nov. 15, 2004; appeared online Nov. 16, 2004; published online Jan. 31, 2005.

\section{Introduction}

With some exceptions, ${ }^{1}$ existing fiber optic refractometers $^{2-4}$ suffer from several disadvantages that limit their effective operation in practical applications. ${ }^{5}$ The most prominent drawbacks of grating-based refractometers are their high sensitivity to temperature fluctuations and the fact that most reported measurements are obtained in transmission. A means of temperature compensation is proposed for use in conjunction with a probe refractometer based on a self-interfering long-period grating (LPG) operating as a Michelson interferometer ${ }^{5}$ (where the constituent grating is both a mode converter and 3-dB coupler).

\section{Michelson Interferometer}

First employed as a high-resolution temperature sensor, ${ }^{6,7}$ the self-interfering LPG structure is manufactured by coating the cleaved end of the host fiber with a reflective metal film at a point beyond the LPG location. Light is guided in both the core and cladding modes after impinging on the LPG, whereupon these modes are reflected at the fiber mirror. When the LPG is encountered once more, a fraction of

0091-3286/2005/\$22.00 @ 2005 SPIE the optical power in the cladding mode recombines with the fundamental core mode. A phase change ${ }^{5,7}$ exists between the re-coupled core and cladding modes because they experience different optical path lengths (due to differing material indices). Thus a characteristic fringe pattern is observed in the reflectance spectrum obtained from this sensor configuration, similar to that found in the transmission of a twin LPG cascade. ${ }^{5-7}$

Highly sensitive external refractive index measurements can be performed if the fiber cavity beyond the LPG is immersed in an analyte. External refractive indices only affect the optical path length of the cladding mode ${ }^{5}$-being the product of its propagation length and effective refractive index. Resulting changes in optical path length difference between the core and cladding modes cause phase shifts in the interference fringe pattern. ${ }^{5}$ The resolution of this sensor can be increased by creating a longer interferometric cavity ${ }^{5}$ (whose length is twice the distance $L$ between the LPG and reflective fiber end-face).

Various LPG-based Michelson interferometers were constructed and subjected to temperatures between $25^{\circ} \mathrm{C}$ and $115^{\circ} \mathrm{C}$. The resulting reflectance spectra were recorded and their phase shifts obtained using Fourier analysis. Two types of host fiber were used, namely: conventional SMF-28 fiber (hydrogen-loaded at 135 bar, $75^{\circ} \mathrm{C}$ for $48 \mathrm{~h}$ prior to grating inscription and annealed at $115^{\circ} \mathrm{C}$ for up to $20 \mathrm{~h}$ after fabrication), and B/Ge co-doped PS1500 fiber from Fibercore. Due to differing core dopants, temperatureinduced phase shifts in reflectance spectra of these Michelson interferometers are of opposite polarity, as shown in Fig. 1. (Please note that a negative phase shift corresponds to a positive shift in the interference pattern.)

\section{Temperature Compensation Technique}

Temperature immunity is obtained by creating a compound interferometric cavity: a piece of the second type of fiber is fusion spliced onto the original host fiber beyond the LPG. Thus the desired interferometer cavity length is divided into two fiber sections joined by a splice, as illustrated in Fig. 2. The length of each type of constituent fiber is calculated according to the ratio of their characteristic phase gradients

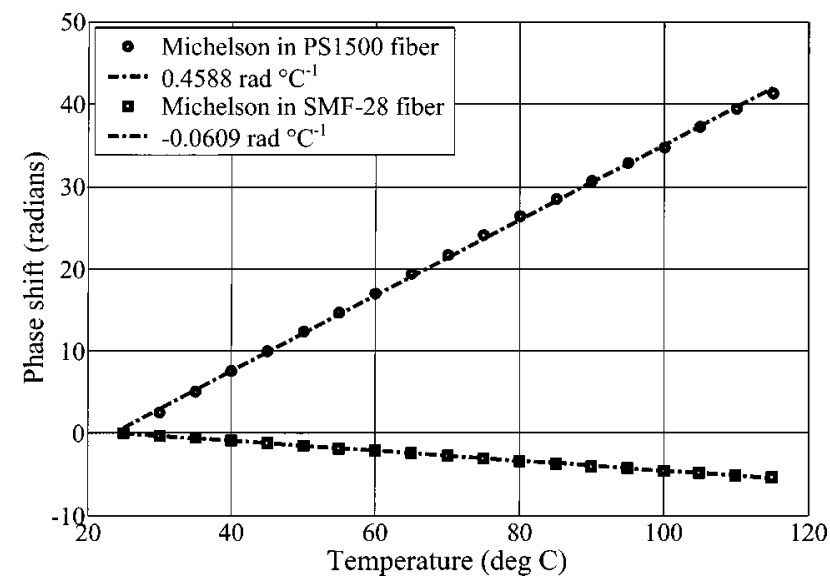

Fig. 1 Temperature characteristics for Michelson interferometers based on LPGs with $\Lambda=464 \mu \mathrm{m}, L_{\mathrm{LPG}}=13 \mathrm{~mm}, L=50 \mathrm{~mm}$ (measured from interference patterns in fifth-order LPG attenuation bands with central wavelengths near $1550 \mathrm{~nm}$ nominally). 
$\mathbf{E}$

$\mathbf{L}$

E

$\mathbf{T}$

$\mathbf{T}$

E

$\mathbf{R}$

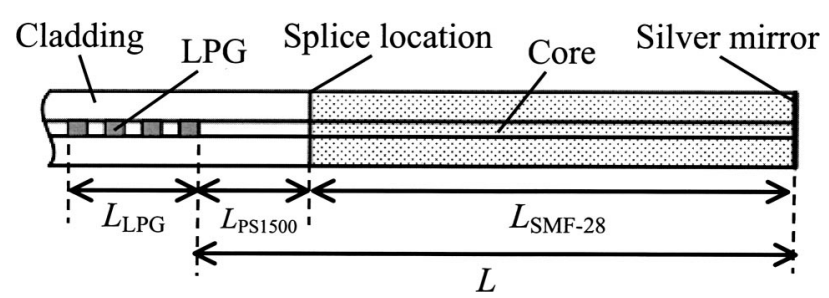

Fig. 2 Structure of proposed temperature compensation method for LPG inscribed in PS1500 fiber with spliced SMF-28 fiber length.

per unit length. For complete temperature compensation, with reference to Fig. 2, it is therefore desired that:

$\left.\left(L_{\mathrm{SMF}-28}\right) \frac{d(\Delta \phi)}{d T}\right|_{\mathrm{SMF}-28}+\left.\left(L_{\mathrm{PS} 1500}\right) \frac{d(\Delta \phi)}{d T}\right|_{\mathrm{PS} 1500}=0$

Compound-cavity Michelson interferometers were manufactured with varying levels of temperature compensation. Figure 3 depicts the phase shifts due to temperature obtained for a long-period grating inscribed in PS1500 host fiber, with several different compound-cavity lengths. The compound-cavity Michelson interferometer containing 107 $\mathrm{mm}$ of SMF-28 fiber was used to perform ambient refractive index measurements using prepared solutions of glycerine in water (calibrated with temperature-controlled Abbe refractometer readings).

The athermal nature of this refractometer was demonstrated by immersing the compound fiber cavity of the Michelson interferometer in a $50 \%$ concentration solution. This analyte was heated and we recorded the resulting phase shifts in reflectance spectra at $5^{\circ} \mathrm{C}$ intervals. The equation below was used to calculate the measured refractive index of the analyte as a function of temperature:

$\frac{\Delta n_{\text {ext }}}{\Delta T}=\frac{\Delta \phi\left(n_{\text {ext }}\right)}{\Delta T} / \frac{\Delta \phi}{\Delta n_{\text {ext }}}$

where $\Delta \phi / \Delta n_{\text {ext }}$ is the slope of refractive index characteristics for the 0 to $80 \%$ concentration range (measured previously at $20^{\circ} \mathrm{C}$ ) at the point corresponding to $50 \%$ glycer-

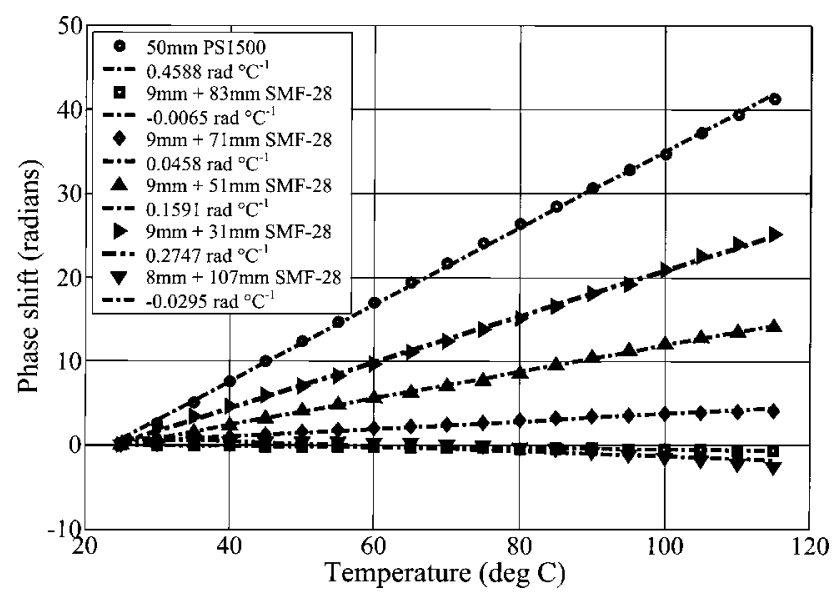

Fig. 3 Measured temperature characteristics for Michelson interferometers with LPG written in PS1500 fiber $\left(\Lambda=464 \mu \mathrm{m}, L_{\mathrm{LPG}}\right.$ $=13 \mathrm{~mm}, L_{P S 1500}=9 \mathrm{~mm} ; 8 \mathrm{~mm}$ ) and spliced SMF-28 fiber lengths.

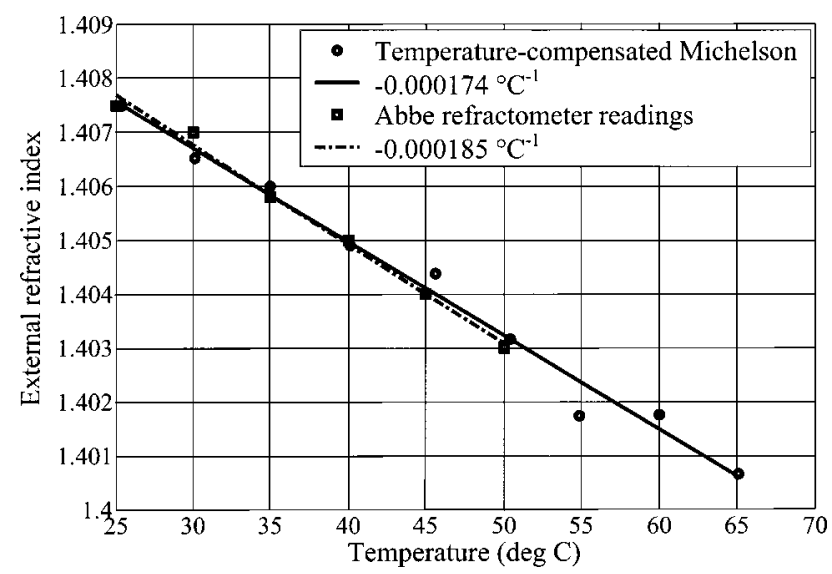

Fig. 4 Temperature-induced changes in external refractive index measured with the temperature-compensated Michelson refractometer and an Abbe refractometer.

ine in water. Phase shifts due to temperature-induced changes in refractive index, $\Delta \phi\left(n_{\text {ext }}\right) / \Delta T$, could be calculated from the results of the experiment described above.

Values of $\Delta n_{\text {ext }} / \Delta T$ determined from Eq. (2) were compared to temperature-dependent Abbe refractometer readings measured for the $50 \%$ concentration solution. Both sets of readings appear in Fig. 4. Numerical gradients of their linear curve fits differ only beyond the fourth decimal place-proof that the temperature-compensated refractometer performs as expected.

\section{Conclusion}

This letter presents a novel means of temperature compensation for use in a LPG-based Michelson interferometer. By constructing a compound interferometric cavity from two types of fiber with different temperature gradients, temperature-induced phase shifts detected in reflectance could effectively be eliminated. The athermal probe refractometer readings are highly satisfactory when compared to Abbe refractometer measurements.

\section{Acknowledgments}

The authors thank ATC (Pty.) Ltd., Marconi Communications South Africa (Pty.) Ltd., Telkom SA Ltd., THRIP, NRF, NLC, and RAU for financial support.

\section{References}

1. Y.-L. Lo and C.-H. Chuang, "Differential optical fiber refractometer based on a path-matching differential interferometer with temperature compensation," Appl. Opt. 40(21), 3518-3524 (2001).

2. G. Laffont and P. Ferdinand, "Tilted short-period fibre-Bragg-gratinginduced coupling to cladding modes for accurate interferometry," Meas. Sci. Technol. 12(7), 765-770 (2001).

3. K. Schroeder, W. Ecke, R. Mueller, R. Willsch, and A. Andreev, "A fibre Bragg grating refractometer," Meas. Sci. Technol. 12(7), 757$764(2001)$.

4. B. H. Lee, Y. Liu, S. B. Lee, S. S. Choi, and J. N. Jang, "Displacements of the resonant peaks of a long-period fiber grating induced by a change of ambient refractive index," Opt. Lett. 22(23), 1769-1771 (1997).

5. P. L. Swart, "Long-period grating Michelson refractometric sensor," Meas. Sci. Technol. 15(8), 1576-1580 (2004).

6. B. H. Lee and J. Nishii, "Self-interference of long-period fibre grating and its application as temperature sensor," Electron. Lett. 34(21), 2059-2060 (1998).

7. B. H. Lee, Y. Chung, W.-T. Han, and U.-C. Paek, "Temperature sensor based on self-interference of a single long-period fiber grating," IEICE Trans. Electron. E83-C(3), 287-292 (2000). 